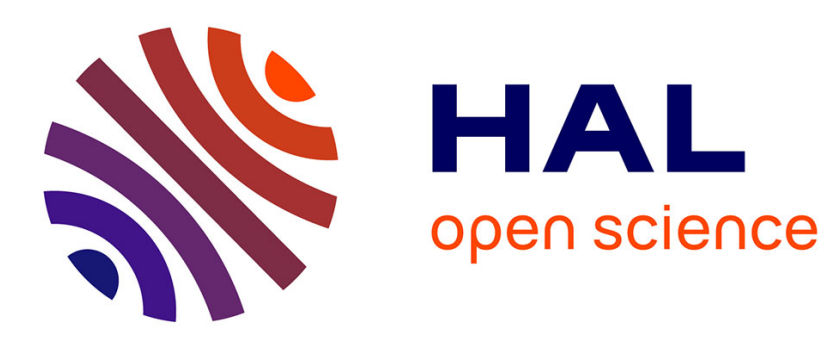

\title{
Société du spectacle et démocratie de la parade dans Cassandre démocrate de Jean Potocki \\ Yves Citton
}

\section{To cite this version:}

Yves Citton. Société du spectacle et démocratie de la parade dans Cassandre démocrate de Jean

Potocki . Jean Potocki à nouveau, Rodopi, pp.75-100, 2010. hal-00847341

\section{HAL Id: hal-00847341 \\ https://hal.science/hal-00847341}

Submitted on 23 Jul 2013

HAL is a multi-disciplinary open access archive for the deposit and dissemination of scientific research documents, whether they are published or not. The documents may come from teaching and research institutions in France or abroad, or from public or private research centers.
L'archive ouverte pluridisciplinaire HAL, est destinée au dépôt et à la diffusion de documents scientifiques de niveau recherche, publiés ou non, émanant des établissements d'enseignement et de recherche français ou étrangers, des laboratoires publics ou privés. 
Yves Citton

(Université de Grenoble - UMR LIRE CNRS 5611)

\section{Société du spectacle et démocratie de la parade dans Cassandre démocrate de Jean Potocki}

Il y a au moins trois façons d'approcher un texte comme Cassandre démocrate. Une étude relevant de l'histoire littéraire peut chercher à réinscrire une telle pièce au sein de ce genre éphémère et très particulier que fut la parade, cette comédie en un acte, mettant en scène la même demi-douzaine de personnages standard (Cassandre, Léandre, Zerzabelle/Isabelle, Gile, le Docteur), destinée originellement à être jouée gratuitement sur les tréteaux des théâtres des Boulevards avec la fonction publicitaire d'attirer le passant et de l'inciter à acheter un billet pour le «vrai » spectacle sur le point de commencer à l'intérieur de la salle. L'aspect a priori déconcertant de la langue («c'est z'ainsi que j'ai appris... qui z'est mon amant»), la minceur d'une intrigue radicalement dénuée de tout souci du vraisemblable et de la psychologie, la dimension essentiellement méta-théâtrale d'une pièce où les discours des personnages ont moins pour référents des types ou des comportements humains que d'autres personnages de théâtre et d'autres discours typés, tout cela - qui pourrait suffire à détourner le lecteur non-averti - s'explique comme une loi de ce sous-genre qui a fleuri entre 1710 et la Révolution ${ }^{1}$. Un tel travail de réinsertion générique et de démêlage des sources et des allusions méta-discursives a déjà été réalisé par Marek Dębowski qui a savamment mis en lumière la façon dont Jean Potocki s'est réapproprié ce genre de la parade pour le farcir des références littéraires les plus diverses et les plus finement ciblées (Rousseau, Mme de Genlis, Jean Larive, etc. $)^{2}$.

Un deuxième type d'études peut soumettre Cassandre démocrate à une lecture relevant de l'histoire des idéologies et des positionnements politiques. On essaie alors de saisir, comme ont commencé à le faire les biographes de Potocki ${ }^{3}$, dans quel état d'esprit, comment et pourquoi un membre de la plus haute noblesse polonaise s'amuse à rédiger et à faire jouer par et pour d'autres aristocrates, durant l'été 1792 dans le château de Łańcut, une série de textes inscrits dans un genre originellement marqué par le théâtre "populaire » de la Foire, mais réapproprié depuis plusieurs décennies pour le plaisir des élites. On est ainsi amené à éplucher les multiples couches de moqueries, d'ironie et probablement de mépris condescendant qui ont pu pousser l'Europe titrée non seulement à adopter les manières de parler poissardes du bas peuple français, mais aussi - dans le cas particulier de Cassandre

\footnotetext{
${ }^{1}$ Pour une bonne présentation de ce genre, voir la préface synthétique proposée par Guy Spielmann à la réédition de quelques-uns de ces textes sous le titre Parades. Le Mauvais Exemple, Léandre hongre, Léandre ambassadeur, Paris, Lampsaque, « Le Studiolo-Théâtre », 2006.

${ }^{2}$ Voir Marek Dębowski, « Originalité des Parades de Potocki », Studies on Voltaire and the Eighteenth Century $\mathrm{n}^{\circ} 265$ (1989), p. 1368-1371; «L'éducation conjugale et sa dérision dans le théâtre polonais des Lumières », in Théâtre et théâtralité. Mélanges offerts à Jean Claude, Presses universitaires de Nancy, 2000, p. 277-284 ; «Parades: le début de l'idée subversive dans l'œuvre de Potocki », ici même.

${ }^{3}$ Voir François Rosset et Dominique Triaire, Jean Potocki. Biographie, Paris, Flammarion, 2004 (pp. 183-242 pour l'époque de la première Révolution et la rédaction des parades) ; Dominique Triaire, Potocki. Essai, Arles, Actes Sud, 1991 ; ainsi que l'introduction rédigée par le même auteur à son édition des Parades, Arles, Actes Sud, 1989, p. 7-16.
} 
démocrate - à parodier les stéréotypes et les idéologèmes du discours révolutionnaire qui était alors à peine en train de prendre forme dans les rue de Paris. Il va de soi que la critique dévastatrice de la rhétorique démocratique mise en scène par une telle pièce est à situer dans un contexte historique (biographique, politique, sociologique, géographique) très précis qui conditionne à la fois le processus de production d'une telle pièce et sa signification. Quoiqu'il s'avère souvent remarquablement proche de nos façons actuelles de percevoir le monde, le conte Jean Potocki ne pouvait bien entendu envisager «la démocratie » qu'à partir de présupposés radicalement différents de ceux qui sont les nôtres aujourd'hui.

En plus de devoir être situé au sein du genre éphémère de la parade, Cassandre démocrate mérite donc également d'être réinscrit dans toute une série de textes parodiant les modes de parole, d'argumentation et de comportement inventés par les révolutionnaires français, ainsi que, plus généralement, dans toute une tradition multi-séculaire de dérision envers le scandale fondamental du principe démocratique : imaginer que le «bas peuple » et la «vile multitude » soient capables de se gouverner eux-mêmes, voilà qui a longtemps suffi à faire rire les élites, et qui déchaînera des torrents de dénonciations de moins en moins amusées au fur et à mesure que la Révolution française se retournera avec plus de rigueur et de cruauté contre l'aristocratie et les divers «ennemis du peuple». En se sens, et même si le texte est écrit avant la radicalisation de 1793, Cassandre démocrate peut être mis en (tête de) série avec les multiples satires de la rhétorique révolutionnaire que connaîtront les dernières années du siècle (comme l'épisode du club de Tornik dans Pauliska ou la perversité moderne de Révéroni Saint-Cyr, ou comme Lioncel, ou l'Emigré de Louis de Bruno).

J'adopterai ici une troisième approche qui écartera explicitement toute prétention historienne pour poser à Cassandre démocrate la question proprement littéraire qui me paraît fondamentale, celle de savoir en quoi ce texte peut encore nous parler aujourd'hui par la seule puissance de sa lettre. Qu'est-ce que, après deux siècles de balbutiements démocratiques, la parade de Potocki peut encore nous apprendre sur la nature, les formes et les limites de nos démocraties actuelles ? En quoi y a-t-il encore une vérité suggestive à tirer de ce texte qu'une lecture superficielle pourrait être tentée de ranger dans un tiroir oublié (et à oublier) de notre histoire littéraire ? Comment le génie littéraire de Potocki réussit-il à transcender les préjugés de la classe, de l'époque et de l'idéologie dans lesquelles baignait l'auteur, pour parvenir à exprimer des intuitions qui conservent à travers les siècles une puissance de révélation constamment renouvelée?

De prime abord, Cassandre démocrate paraît se conformer pleinement à la vision qu'un aristocrate polonais pouvait adopter sur la Révolution française. La pièce met en scène un conflit entre un petit commerçant partisan de la révolution démocratique (Cassandre, le père) et un défenseur des droits de la noblesse (Léandre, l'amant), entre lesquels se trouve prise Zerzabelle (la fille à marier), que Cassandre souhaite faire épouser son commis (Gile), auquel il a remis sa petite entreprise (dépérissante) de vente de fromages. Comme de juste, le prétendu démocrate y est ridiculisé de part en part : il s'approprie un vocabulaire politique à la mode qu'il détourne de son objet premier et auquel il paraît ne rien comprendre («je vais procéder à l'appel nominal » ${ }^{4}$ signifiant simplement qu'il s'apprête à appeler sa fille vers lui) ; le récit de vie par lequel il se présente au public est un tissu de mensonges, à travers lesquels l'orphelin analphabète se déclare "né au sein d'une famille honnête », et avoir reçu «une éducation excellente » lui donnant l'occasion de «se distingu[er] de [s]es compagnons d'étude» (II, 47); malgré son recours exagéré au lexique démocratique, il prétend se comporter en petit despote envers sa fille qu'il donne en mariage sans la consulter et sans

\footnotetext{
${ }^{4}$ Jean Potocki, Cassandre démocrate, scène I, in Euvres, éd. François Rosset et Dominique Triaire, Louvain, Peeters, 2004, tome III, p. 46 (noté dorénavant I, 46). Pour faciliter la lecture des citations, j’en ai modernisé la ponctuation et l'orthographe.
} 
même la laisser parler («Taisez-vous », «Taisez-vous donc», II, 47); ses velléités despotiques sont d'autant plus ridicules qu'elles n'ont aucun effet sur une fille bien résolue à n'en faire qu'à sa tête, et à épouser son amant aristocrate Léandre plutôt que le valet Gile ; à peine commence-t-il à être rossé par Léandre que Cassandre retourne sa veste et lui offre sa fille en mariage, tout en prétendant par ce geste «sauver la France» de la «contrerévolution » $(\mathrm{V}, 51)$. Le bourgeois démocrate est donc peint sous les traits traditionnellement attribués par les élites à «la lie du peuple»: ignorant, stupide, prétentieux, menteur, lâche, veule, opportuniste, volage et naturellement porté vers la tyrannie - bref, incapable de se gouverner soi-même. On peut facilement y sentir la condescendance du noble envers «ces grands enfants que l'on appelle peuples $»^{5}$, et on imagine sans peine les rires satisfaits et les sourires méprisants de la coterie patricienne réunie au château de Łańcut devant un tel tableau (très convenu) de la plèbe révolutionnaire démocrate.

Toute la seconde moitié de cette petite pièce retourne toutefois le projecteur pour faire apparaître l'aristocrate Léandre aussi ridicule que le démocrate Cassandre. Nourrissant sa superbe de jurons et de coups, le jeune homme se déchaîne contre les «vils plébéiens » dont l'assemblée «a défendu de mettre des armoiries aux portières des carrosses », contre «la populace infâme » qui a «décrété qu'il ne serait pas permis de mettre ses armes sur la porte de son hôtel », et contre les «marauds » qui ont «défendu les livrées » (V, 50), mais son emportement tombe aussi ridiculement à plat que les mensonges prétentieux de Cassandre puisque, comme Gile ne manque pas de le souligner à chaque réplique, Léandre n'a ni carrosse, ni hôtel, ni domestique. Cet aristocrate, fier de ses droits traditionnels mais issu du plus bas étage et vivant sans le sou, s'avère tout aussi tyrannique que le démocrate Cassandre, puisque lui non plus ne tolère pas la moindre réplique («Taisez-vous, lie du peuple » V, 50) et ne se fait entendre par des coups distribués très généreusement à tout ce qui passe à sa portée - y compris sa dulcinée : «Mamzelle, si ce n'était la chevalerie dont je fais profession, $\&$ qui rend votre personne inviolable, je vous traiterais comme ces deux marauds » $(\mathrm{V}, 51)$. À la veule multitude ne fait donc face qu'une brute épaisse entichée de titres et de privilèges devenus sans objet. Au-delà de leurs préjugés de caste, les hôtes du château de Łańcut semblent donc avoir été disposés à se moquer de leurs semblables (de la petite noblesse, du moins) aussi bien que de ceux qui menaçaient leur statut au nom du peuple et de l'égalité.

Sur ce registre, on pourrait rapprocher utilement Cassandre démocrate de la Seconde lettre sur l'histoire de notre temps parue le 29 février 1792 dans le Journal Hebdomadaire de la Diète, soit six mois avant la rédaction de la parade. Après avoir rencontré Mirabeau et Lafayette lors de son séjour de novembre 1790, au cours duquel il avait été invité au club des Jacobins et à l'Assemblée nationale ${ }^{6}$, Potocki revient sur son plus récent voyage à Paris de novembre et décembre 1791 pour relever que «l'esprit de parti y est poussé à l'excès, et que tout ce qui porte le caractère de la modération ou de l'impartialité est aussitôt rejeté par les deux partis ». Or il identifie ces partis sous des termes qui annoncent clairement les personnages de la parade. Relevant que leur seul point d'accord consiste en leur admiration commune pour la révolution polonaise, il ajoute que, même sur ce terrain d'entente, ils parvenaient se disputer : « les Aristocrates disaient que nous leur appartenions, parce que nous avions conservé la noblesse, et les Démocrates nous revendiquaient, parce que nous avions admis le Tiers ». Et d'ajouter une remarque qui montre à quel point le choix de ces termes est chargé d'une conscience hautement réflexive :

Mais, Monsieur, puisque je viens de me servir des noms d'Aristocrates et de Démocrates, je crois devoir répondre à la communication que vous m'avez faite d'un

\footnotetext{
${ }^{5}$ Jean Potocki, Lettre du 17 janvier 1794 à Madame de Staël in Euvres, éd. de François Rosset et Dominique Triaire, Louvain, Peeters, 2006, tome v, p. 35.

${ }^{6}$ Voir sur ces points la Lettre du 23 décembre 1790 à Séverin Potocki in Euvres, v, p. 23 sqq.
} 
billet de Mr. L'A[bbé] P[iattoli] où cet ami commun vous invite à ne jamais donner le nom d'Aristocrates à nos mécontents, parce qu'il pourrait en résulter que l'on donnerait le nom de Démocrates au parti opposé, ce qui serait absolument faux ${ }^{7}$.

Alors que Potocki se donnera la peine (bien peu méthodique) de préciser les «nouvelles définitions » qu'il convient d'attribuer à des termes comme «Ambition », «Patriotisme », « Bon citoyen », «Honnêtes gens » ou «Coquins » dans la Quatrième lettre sur l'histoire de notre temps parue le 14 mars 1792, il ne fournira pas celles qu'il avait pourtant annoncées à propos des «Démocrates » et des «Aristocrates ». On peut imaginer que la parade lui offre l'occasion (opportunément ludique) de compléter ce travail de redéfinition qui mêle inextricablement les registres politiques, comiques et satiriques. Outre une condamnation générale de l'esprit de parti et de l'intransigeance aveugle qui caractérise les agents politiques de la période révolutionnaire, le «sens » de la pièce paraît alors relativement clair: si l'intrigue (minimale) finit, comme il se doit, sur le happy end d'un mariage accepté par Cassandre entre Zerzabelle et Léandre, c'est seulement parce que la force brutale (et aveugle) de la noblesse a remis sur le droit chemin de la soumission les prétentions ridicules d'une populace lâche et infâme qui se gargarisait ridiculement d'un lexique politique dévoyé auquel personne ne comprend plus rien. Une analyse aussi cynique ne manquait sans doute ni de réalisme ni d'attrait pour les aristocrates réunis autour du comte Jean Potocki - et elle correspond à des interprétations assez largement répandues parmi l'Europe titrée de l'époque, et dont on peut voir quelques traces émerger ailleurs dans l'œuvre de Potocki ${ }^{8}$. Il serait sans doute sage d'y reconnaitre «la» signification (historique) de Cassandre démocrate, et d'arrêter ici les frais d'une interprétation qui ne peut se prolonger sans risquer de partager le ridicule impertinent de Cassandre farcissant du lexique spécialisé de la démocratie une banale affaire matrimoniale...

Car, bien entendu, cette piécette relève de la plaisanterie, et non de l'analyse historique ou du traité politique. De même que le Manuscrit trouvé à Saragosse avait sans doute pour fonction première, dans l'esprit de son auteur, de divertir, et que c'est sur le mode d'un plusde-sens que nous pouvons aujourd'hui en tirer des enseignements d'ordre philosophique ou anthropologique, de même les parades ont-elles évidemment pour but premier de faire rire, et non d'articuler une théorie politique. Ce sont toutefois bien les éléments d'une théorisation de la démocratie que je souhaite dégager de ce texte bref, théorisation que je résumerai et systématiserai en neuf thèses succinctes, ordonnées de la plus triviale à la moins intuitive - en prenant (trop) au sérieux cette plaisanterie que j'érigerai (par un forçage interprétatif avoué pour tel) au statut de «modèle »: à quoi ressemblerait la démocratie si les paroles, les comportements et les mésaventures de Cassandre devaient lui servir de révélateur?

1. La gouvernementalité démocratique participe d'une "révolution» (moderne) qui renverse les valeurs traditionnelles (fondées sur la continuité du sang et du titre) en de nouvelles valeurs se réclamant d'un processus institutionnel d'éducation et de décision politique collective.

\footnotetext{
${ }^{7}$ Jean Potocki, Seconde lettre sur l'histoire de notre temps in Euvres, III, p. 319.

${ }^{8}$ Pour ces affleurements d'un mépris pour la plèbe incapable de se gouverner elle-même rationnellement, voir par exemple Euvres, III, p. 271-272 ou p. 311. Rien n'étant jamais simple chez Potocki, il est également vrai que le très riche Essai d'aphorismes sur la liberté de 1790, en même temps qu'il affirme des vues qui ne peuvent nous apparaître rétrospectivement que « racistes », décrit l'émancipation en termes d'un art subtil grâce auquel les mœurs des peuples interagissent circulairement avec les initiatives législatives (ou normatives), selon des cercles qui peuvent être souvent vicieux mais parfois vertueux (CEuvres, III, p. 305-311). Pour un écho avec des réflexions contemporaines sur ces sujets, voir l'article de Marc Maesschalck, «Normes de gouvernance et enrôlement des acteurs sociaux » dans la revue Multitudes, n $^{\circ} 34$ (automne 2008).
} 
Le combat (de fantoches) qui confronte Cassandre à Léandre oppose bien deux systèmes de valorisations et de références. Le démocrate se réclame certes "d'une famille honnête », mais il situe cette honnêteté dans le fait que ses parents «ne négligèrent rien pour [lui] donner une éducation excellente »; il tire sa fierté de s'être «distingu[é] bientôt de [s]es compagnons d'étude », de s'être entièrement voué aux «soins de l'éducation» de sa fille et d'avoir «toujours été démocrate », ce dont il témoigne en se restreignant à ne faire que ce qui est « dans la constitution», à ne se fier qu'à la «pluralité assurée » et à soumettre toutes ses décisions aux formes de «la motion», du «veto suspensif», de «l'ordre du jour» et de «l'appel nominal» (I, 46 \& II, 47-49).

L'aristocrate, de son côté, ne se fie qu'à son épée, qu'il entend «passer au travers de l'assemblée nationale », il ne respire que le mépris du bas peuple, dont il se distingue par ses armoiries, ses armes et ses livrées, autant de marques d'une identité héritée par un lignage qui suffit à justifier sa supériorité. Face à «l'insolence» de «l'impertinent raisonneur » et des « vils plébéiens » qui refusent de reconnaître cette supériorité de droit, il « voit qu'une contrerévolution est nécessaire », il se résout à «l'opérer ici, ce sera toujours autant de fait », et il entreprend aussitôt de rosser tous ceux qui passent à portée de ses coups (V, 49-51).

La pièce se situe explicitement au lendemain d'un renversement des valeurs traditionnelles : alors que la déchéance consistait auparavant en ce qu'un noble épouse une roturière, le scandale tient désormais à ce que la fille d'un bourgeois fromager désire « épouser un gentilhomme» qui «porte une épée » (II, 49). À l'ordre ancien assurant la supériorité du sang et de la bravoure combative, une révolution a bel et bien substitué un ordre nouveau caractérisé par le culte de l'égalité juridique, le respect des processus parlementaires, la distinction par le mérite personnel et l'éducation - ordre nouveau auquel on ne peut s'opposer que par une contre-révolution.

2. La gouvernementalité démocratique est une affaire d'entrepreneurs commerçants, dont l'existence est vouée à assurer la meilleure gestion de leur capital (matériel, financier, humain, symbolique).

Durant l'exposition qui lui donne l'occasion de peindre le tableau flatteur de sa vie et de sa trajectoire sociale, Cassandre le démocrate précise qu'au moment de «choisir un état », il «préfér[a] le commerce, qui est comme l'on dit l'âme des états commerçants », se vouant désormais à «émettre des fromages pour la consommation du bon peuple, que ses ennemis n'ont point assez calomnié » (II, 47). Au-delà de cette vocation à se mettre au service de la production et de la circulation des marchandises, le démocrate apparaît surtout comme un individu qui gère toutes les dimensions de son être sur le mode de l'investissement, de la transaction et de l'échange. Lorsque «la révolution fit tomber tout le commerce de la France » et que «la consommation de [s]es fromages diminuait tous les jours », Cassandre s'avisa non seulement de "proposer à l'assemblée l'émission de cent millions d'assignats », mais surtout il se «résolu[t] à abandonner à [son commis] Gile un commerce qui ne fait plus la circulation d'un dividende favorable »; tout laisse cependant supposer que la remise de sa boutique à fromages s'inscrit dans une transaction plus large, au sein de laquelle ce don a valeur de dot, et constitue ainsi à la fois un placement de sa fille et une assurance-vieillesse : « en même temps, je lui fais un présent plus rare quoique moins commun, je lui donne en mariage ma chère Zerzabelle » (II, 48).

Pour le démocrate, les «trois commerces » de Montaigne n'en font plus qu'un : amour, amitié, mariage, filiation, finance, offre et demande ne forment plus qu'un vaste réseau de transactions au sein duquel tout peut communiquer avec n'importe quoi. Cassandre ressemble bien à la figure de «l'entrepreneur de soi-même» que Michel Foucault a esquissée à l'horizon de l'anthropologie néolibérale dans ses cours au Collège de France des années 19771978 : un homo oeconomicus qui gère toutes les dimensions de son existence en termes de 
capital à investir de façon à en maximiser les revenus. On passe ainsi avec une parfaite fluidité de la vente de fromages au mariage de sa fille, d'une opération financière sur les assignats à une promotion de sa réputation, de l'amour pour ses enfants au soin de son patrimoine - chaque individu ne constituant plus pour lui-même qu'une forme particulière de capital (le capital humain) à faire fructifier de la manière la plus judicieuse et la plus prudente.

Une telle prudence explique la hantise qu'éprouve l'entrepreneur démocrate à l'idée de s'exposer à quelque forme que ce soit de violence destructrice. Dès lors que l'individualisme possessif fait coïncider l'individu avec ses possessions, et dès lors que la grande affaire d'une vie est de savoir faire fructifier au mieux toutes les dimensions de son capital, l'entrepreneur devient un lieu de tension paradoxale entre une prise de risque qui définit son essence (du point de vue d'une théorie économique déjà mise en place un demi-siècle avant Potocki par Cantillon et les physiocrates), et une profonde aversion envers toute violence pouvant entraîner la perte ou la destruction de ce capital qui constitue son être même (d'où la multiplication progressive des mécanismes assuranciels). Le valet pusillanime, prêt à toutes les conciliations les moins honorables, a toujours été l'autre face du chevalier d'industrie.

Telle est bien l'image (ridiculisée) qu'en reflète ici Cassandre. Après avoir "opéré une contre-révolution » par une pluie de coups appliqués aux épaules de Gile, l'aristocrate s'est mis à rosser le démocrate qui, loin de songer à répondre à cette violence sur le mode du combat, ne cherche d'abord qu'à l'esquiver, puis à la faire tourner au profit de son capital (symbolique) :

CASSANDRE: L'on avait bien raison de dire que rien ne serait plus fatal qu'une contre-révolution, \& je puis en parler à présent par expérience ; cependant il me reste un moyen d'empêcher que cela n'aille plus loin : ce Léandre est amoureux de ma fille, je m'en vais la lui offrir en mariage, à condition qu'il renoncera désormais aux contrerévolutions, z'ainsi j'aurai une occasion de sauver la France, \& de mériter une couronne civique, que je ne manquerai pas de faire mettre dans les Gazettes. (V, 51).

C'est bien la même logique entrepreneuriale qui poussait Cassandre à «donner en mariage » Zerzabelle à Gile dans la scène II, et qui le pousse ici à revoir ses plans d'investissements pour l' «offrir en mariage » à Léandre. Dans les deux cas, la chair de ma chair (mes épaules soumises aux coups d'un violent, ma fille en âge de mariage, mon entreprise nourrie de ma sueur, mon image publique garante de ma crédibilité à venir) entre dans un commerce généralisé aux permutations infinies, dont toute ma vie (prudente) se passe à anticiper les retournements, à calculer les opportunités et à éviter les écueils.

\section{La gouvernementalité démocratique recouvre les rapports de force et de violence} d'un vernis fragile de consentement.

Contrairement à ce que j'ai laissé entendre jusqu'à présent, la violence (destructrice) ne constitue toutefois pas une réalité extérieure au monde de la démocratie entrepreneuriale. $\mathrm{Si}$, dans les gestes mis en scène par la parade, c'est bien le brutal Léandre qu'on voit « renverser tous les meubles » $(\mathrm{IV}, 49)$, « jeter à terre » et « rosser » les autres personnages $(\mathrm{V}, 51)$, et si le pusillanime Cassandre paraît plier sous cette violence en ne cherchant qu'à s'en concilier les bonnes grâces, le discours du démocrate révèle dès la première tirade à quel point les démocraties ne font souvent que recouvrir de formes consensuelles des menaces et des rapports de force qui constituent le ressort caché du consentement qu'elles génèrent :

CASSANDRE : Je suis seul, profitons de ce monologue favorable pour faire venir ma fille, \& lui faire la motion d'épouser un mari de ma main, qui sera l'époux que je lui destine. Je ne doute point de son assentiment, \& si par hasard elle voulait user du veto suspensif, je lui donnerais cent coups de pieds dans le ventre, qui sont une pluralité 
assurée \& donnant ainsi au pouvoir exécutif une énergie momentanée, je vais procéder à l'appel nominal. Zerzabelle, Zerzabelle ! (I, 46)

Cette parodie d'énergétique politique à la Montesquieu double son effet comique d'un effet révélateur quant à l'esprit des lois (et des décrets exécutifs) qui contiennent la multitude en régime démocratique : sous la liberté d'expression, on voit périodiquement réapparaitre les «cent coups de pieds » et de bâtons de la police anti-émeute; sous la liberté des contrats (d'emploi), on sent souvent peser les exigences de la faim « dans le ventre », les angoisses des loyers, des traites et des fins de mois difficiles, qui motivent les démocrates les moins bien lotis à donner «leur assentiment » à des formes de vie qui ne font pas vraiment pour eux l'objet d'un choix... Sous le triomphe glorieux de l'homo democraticus réglant le sort de ses gouvernants selon le bon plaisir majoritaire de ses inclinations électorales, sous les bastonnades momentanées que le pouvoir exécutif impose aux épaules de ses opposants trop énergisés, la démocratie marchande repose bien sur une pluralité assurée de contraintes, qui restent généralement en deçà de la violence ouverte, mais qui nourrissent son fonctionnement quotidien de leurs menaces latentes.

Il est intéressant à cet égard de voir Potocki inscrire, sans nécessité dramaturgique particulière, la frontière extérieure de la violence esclavagiste des colonies américaines au sein de son portrait de la démocratie commerçante parisienne. Cassandre, que «[s]on goût portait aux établissements éloignés », a cependant retreint ses pérégrinations du «faubourg St Marceau » aux «bords de la Seine », sous l'effet d'un noble scrupule moral qui l'a conduit à renoncer aux profits du commerce colonial :

CASSANDRE: Un autre que moi peut-être eût fait cultiver ses fromages en Amérique, mais je ne voulus point d'un or arrosé du sang de malheureux qui, pour n'être pas plus noirs que vous, ma chère Zerzabelle, n'en sont pas moins vos frères. (II, 47)

En un «siècle des philosophes » dont un ouvrage récent montre qu'ils ont eu fortement tendance à scotomiser la réalité esclavagiste de leur vision du monde ${ }^{9}$, cette irruption gratuite de la traite négrière dans le discours de Cassandre, pour multi-comique qu'elle soit ${ }^{10}$, fait écho aux «cent coups de pieds dans le ventre » du monologue initial en suscitant le fantôme des chaînes, des brimades, des mutilations et des massacres que les entrepreneurs de commerce, avec tout l'appui du pouvoir exécutif, imposent par la force la plus brutale et la violence la

\footnotetext{
${ }^{9}$ Christopher L. Miller dans The French Atlantic Triangle. Literature and Culture of the Slave Trade (Durham \& London, Duke University Press, 2008) soutient qu'à quelques exceptions près, les philosophes les plus importants des Lumières françaises «n'ont pas trop dérangé le commerce d'esclaves : Rousseau, concentré sur des problèmes européens, l'a ignoré presque entièrement, et Voltaire en a fait l'objet d'investissements, tout en faisant des commentaires spirituels et ironiques sur l'inhumanité de l'ensemble. L'application à l'Afrique de ces notions des Lumières que sont la liberté et l'auto-détermination auront encore longtemps à attendre, jusqu'à ce que les mouvements d'indépendance s'emparent de l'idée d'auto-détermination et se l'approprient au XX ${ }^{\mathrm{e}}$ siècle. Au XVIII ${ }^{\mathrm{e}}$ siècle, le commerce d'esclaves et l'esclavage ont persisté pour le profit d'une classe qui se considérait cultivée » (p. 81, ma traduction).

${ }^{10}$ La densité comique obtenue dans la plupart des répliques de cette pièce par la superposition de multiples niveaux de dérision est remarquable, et en dit long sur le talent littéraire de Potocki. Pour ne prendre que le cas de ces quelques mots consacrés au commerce esclavagiste, on peut identifier au moins $1^{\circ} 1^{\prime}$ absurdité évidente d'importer des fromages d'Amérique étant donné la lenteur des transports de l'époque, $2^{\circ}$ le ridicule d'un goût pour les établissements lointains qui se borne à un déménagement de quelques centaines de mètres au cœur de Paris, $3^{\circ}$ le contresens d'une langue qui fourche pour peindre en noire la blanche Zerzabelle, $4^{\circ} 1^{\prime}$ hypocrisie d'un manque d'esprit d'aventure glorifié rétrospectivement au titre d'un scrupule moral - à quoi l'on verra bientôt s'ajouter une cinquième couche (dénonçant un conformisme décervelé).
} 
plus sauvage à leurs «frères » moins blancs qu'eux, aux périphéries de la démocratie européenne.

4. La gouvernementalité démocratique rencontre sa limite dans la résistance que lui opposent les convenances et les répugnances des sujets à gouverner - convenances et répugnances qui constituent l'énergie réelle dont vit la démocratie radicale.

Malgré la menace des cent coups de pieds dans le ventre, Zerzabelle refuse son assentiment au mariage avec Gile prévu par son père - et se voit provisoirement déshéritée à la fin de la scène IV. Si l'on veut voir en la démocratie (radicale) une force (kratos) de résistance exercée par le bas peuple (démos) à toute forme de pouvoir tyrannique imposé par «le haut », c'est en réalité du côté de Zerzabelle, et non de Cassandre, qu'il faut repérer la figure du démocrate. Sous les irruptions momentanées et localisées de violence ouverte (coups dans les ventres, gaz lacrymogènes, emprisonnements, exécutions, ou encore évictions et licenciements), la démocratie repose au quotidien sur la reproduction et l'acceptation tacite d'un régime de convenances. Comme l'a mis en lumière de façon paradoxale le Discours sur la servitude volontaire de La Boétie, et comme l'a développé la tradition politique aujourd'hui identifiée au spinozisme, toute forme de pouvoir politique stable se nourrit d'une obéissance fondée sur le fait que la multitude des sujets croit trouver davantage d'intérêt à se plier aux consignes du pouvoir en place qu'à s'y opposer ouvertement - en fonction de quoi l'on peut dire que toute forme de gouvernement est (de façon radicale: dans ses racines mêmes) démo-cratique. Au vu de cette approche, la limite du pouvoir politique n'est donc pas tant à situer dans la vertu d'un texte (constitution, code de lois, etc.) que dans un certain régime de circulation d'affects : les sujets veulent bien obéir aux lois aussi longtemps qu'ils s'imaginent que l'ordre politique en place convient (en gros) à leurs aspirations, mais ils sont toujours prêts à refuser d'accorder cette obéissance (voire à recourir à la violence antigouvernementale) dès lors que les mesures proposées leur répugnent et causent leur indignation - les démocraties électorales ne faisant de ce point de vue que soumettre les gouvernants à la mesure formelle et régulière de la circulation des affects qui constitue le fondement de la démocratie radicale ${ }^{11}$.

C'est précisément dans ces termes que Zerzabelle réplique au premier projet de mariage formé par son père :

ZERZABELLE: [...] j'ai l'honneur de vous répondre avec ma douceur accoutumée que si l'époux me convient, je le garderai, mais que les coups de pieds ne m'ayant jamais convenu dans le ventre, je vous les rendrai avant que vous me les ayez donnés. [Son père lui ayant demandé de s'asseoir pour l'écouter :] je sais que le devoir d'une fille soumise est d'obéir, lorsqu'elle n'y a point de répugnance, \& je vais m'asseoir. (II, 46)

Fidèle à ses principes, Zerzabelle s'assied, puisque cet ordre lui convient, mais refuse d'épouser Gile, puisque ce commandement lui répugne - ne voulant «épouser que Monsieur Léandre, qu['elle] aime \& qui z'est [s]on amant depuis le jour où il s'est offert à [s]a vue » (II, 48).

La réponse de Cassandre à un tel refus d'obéissance est doublement révélatrice du fonctionnement politique d'une démocratie (commerçante). D'une part, il est porté à ne voir dans le refus d'obéissance de sa fille que l'effet de menées démagogiques : « vous le prenez là sur un ton bien démagogue » (II, 48). D'autre part, et dans la même foulée, il se retient de recourir à la violence ouverte, au nom du respect du texte de la constitution, mais impose aussitôt une sanction d'ordre économique : «si les coups de pieds étaient dans la constitution,

\footnotetext{
${ }^{11}$ Sur tous ces points, je renvoie à Yves Citton et Frédéric Lordon (éd.), Spinoza et les sciences sociales. De la puissance de la multitude à l'économie des affects, Paris, Éditions Amsterdam, 2008.
} 
tu n'en recevrais que de ma main. Mais je sens que je m'écarte de l'ordre du jour. Je lève la séance, je t'abandonne à ton Aristocrate, \& je te déshérite » (II, 49).

Le quiproquo de la réplique de Zerzabelle - «Non, mon père, je ne le prends point sur un ton décalogue »- fait habilement le lien entre les deux réactions de Cassandre : le gouvernant démocrate résiste difficilement à la tentation de qualifier de « démagogue » (ou de «populiste ») toute affirmation de désir ou de répugnance en provenance de la multitude, dès lors qu'elle contrevient à la lecture (forcément intéressée) qu'il propose du « décalogue » législatif ou constitutionnel en charge de réglementer la vie démocratique. Et au-delà d'une telle dénonciation de démagogie, s'il n'entreprend pas d'imposer par la force un bien-être dont la multitude (aveugle) ne veut pas, il s'empressera de menacer de rendre la vie économiquement impossible (au nom de la croissance du PIB et de la compétition internationale) à quiconque se refusera à partager son interprétation du décalogue.

\section{La démocratie est une affaire de régime rhétorique avant d'être une affaire de mode} de gouvernement.

En deçà de ces considérations «de substance »sur le fonctionnement de la politique démocratique, ce que la parade de Potocki met surtout en lumière (comique), c'est d'abord un certain régime de circulation, de déformation, de torsion et de déplacement de la parole et de la nomination. On a déjà vu dans les citations précédentes à quel point le « démocratisme »de Cassandre consistait avant tout à nommer par des termes tirés du lexique politico-législatif des actions relevant en réalité de ses gestes quotidiens et de ses intérêts purement privés («faire la motion d'épouser un mari », «procéder à l'appel nominal », «lever la séance »). On aura également senti que le comique repose généralement sur les quiproquos qui intervertissent les syllabes ou les étiquettes dans cette valse parfaitement immaîtrisée de nomination pseudopolitique - démagogue devenant décalogue, démocratique, Démocrite, aristocrate, Erostrate, décrété, décrotté, la «contre-révolution » nommant une bastonnade, et «les deux chambres » désignant la cuisine \& l'alcôve de la maison de Cassandre.

Le comique de la parade repose essentiellement sur de tels jeux de déplacements (lexicaux, perlocutoires), de même que sa suggestivité théorique repose sur les déplacements conceptuels qu'invitent à opérer ces déplacements verbaux. Avant de les observer plus en détails, il convient toutefois de mesurer leur impact d'ensemble, qui est de redécrire la démocratie comme un mode de s'exprimer tout autant (voire davantage) que comme un mode de gouverner. Dans la mesure même où la pièce met en scène avec Cassandre un démocrate ridiculement indigne de l'idéal démocratique - comme elle met en scène avec Léandre un aristocrate notoirement indigne de l'idéal de la noblesse -, elle nous conduit à concevoir la démocratie comme un régime rhétorique de gouvernementalité largement indépendant des convictions, des finalités et des pratiques concrètes des acteurs qui en jouent le jeu.

6. Le régime rhétorique de gouvernementalité démocratique fait que nul n'y sait vraiment de quoi il parle ni ne peut s'en tenir à la parole qu'il fait mine de donner.

La parade nous plonge dans un univers où nul ne coïncide pleinement avec l'identité dans laquelle il essaie de se (faire) reconnaître. De par le ridicule qui doit, par une loi du genre, recouvrir tout personnage, le démocrate et l'aristocrate sont voués à paraître tragicomiquement en retrait par rapport à l'idée qu'on voudrait se faire de leur statut et de leurs prétentions. Dans la mesure où la démocratie se conçoit comme un lieu de négociation collective (et donc de compromis, d'alliances et de flexibilité), chacun s'y voit condamné à devoir faire ce qu'il s'était promis de ne pas faire, comme l'illustre bien l'attitude de Léandre envers Cassandre à la fin de la pièce : « votre alliance est de nature à ce qu'un gentilhomme ne devrait pas seulement la prendre avec des pincettes, mais les appâts de votre fille me décident \& je consens à me mésallier ». Sur la base de cette première compromission se 
déroule une négociation au cours de laquelle Léandre promet «de ne plus songer à une contre-révolution », tandis que Cassandre «accorde les deux chambres » à son ennemi politique, devenu son beau-fils et pouvant désormais s'installer dans la cuisine et l'alcôve de sa maison fromagère. Le démocrate fait certainement rire mais il n'a nullement tort de dire que « grâce à [s]on esprit conciliatoire, la contre-révolution n'a pu s'effectuer» $(\mathrm{V}, 52)$. La véritable contre-révolution dans une démocratie parlementaire ne consiste pas en un changement de gouvernement ou d'orientation politique, mais en une suspension de l'esprit conciliatoire (par un coup d'État militaire ou par l'instauration d'un état d'exception) - soit en l'instauration d'une parole qui refusera de se plier aux formes du compromis.

S'il est vrai qu'en démocratie l'agent politique ne peut s'engager que conditionnellement à vouloir faire demain ce qu'il (se) promet aujourd'hui, il n'est guère étonnant de le voir ne pas savoir non plus très précisément sur quoi portent sa parole et ses promesses actuelles. Tous les déplacements dont se nourrit le comique de la parade (les quiproquos, les confusions, les malentendus) participent d'un débordement inévitable de la parole envers l'action (et de l'action envers la parole) au sein du processus démocratique. Parce qu'il s'agit d'un processus par essence collectif et orienté vers le futur (un désir de prospérité à assurer pour l'avenir, au sein de conditions qu'il est impossible de connaître ni de prévoir de façon précise et certaine), aucun agent individuel ne peut savoir exactement ce qu'il dit en offrant les solutions législatives qui lui semblent les plus désirables. La rhétorique démocratique est donc fatalement immaîtrisée - truffée d'approximations et d'erreurs d'estimation, hantée de contradictions, qui n'apparaîtront pleinement qu'avec le recul apporté par (les ratages de) l'expérience à venir.

Les agrammaticalités et les paralogismes dont la parade farcit ses dialogues ne font qu'exacerber les imprécisions, les omissions, les illusions ou les impostures dont se nourrit toute parole politique en régime démocratique : prétendre faire «un présent plus rare parce que moins commun»(II, 48), affirmer que les esclaves africains ne sont «pas plus noirs que »Zerzabelle (II, 47), ou traiter cette dernière de «fille-marâtre » (II, 49) ${ }^{12}$, tout cela est certainement plus drôle mais pas forcément moins absurde que de prétendre mesurer le bienêtre commun à la seule croissance du PIB, que de traiter les immigrés comme une menace pour la prospérité nationale, ou que de disqualifier a priori tout programme impliquant une hausse d'impôts. Dès lors qu'on analyse à la loupe le parcours argumentatif des discours politiques qui circulent dans nos médias, ils prennent rapidement l'allure de ces «coq-àl'âne » et de ces «amphigouris » que Zerzabelle dénonce plusieurs fois dans le bavardage de son père.

7. Le régime rhétorique de gouvernementalité démocratique impose des formes et des contenus de discours convenus, indépendants de la vérité éprouvée par le sujet parlant.

Alors que les absurdités alignées par Léandre tiennent à ce qu'il se laisse emporter par sa défense furieuse des droits ancestraux de la noblesse, il apparaît vite comme évident que Cassandre le démocrate ne croit pas un seul des mots qu'il profère. Les mensonges éhontés dont il alimente son autobiographie ne participent ni d'un emportement illusionné, ni même d'un désir de faire accroire à son interlocuteur une contre-vérité dont il aurait intérêt à le persuader, mais semblent relever d'un besoin bizarre de proférer des choses auxquelles il sait pertinemment que son interlocuteur ne croira pas, mais qui doivent néanmoins être affirmées pour la forme. Lorsque Zerzabelle lui rappelle qu'il est orphelin et ne sait ni lire ni écrire,

\footnotetext{
${ }^{12}$ Potocki utilise un mode de ridiculisation similaire (de type nonsensical) dans quelques-uns de ses textes ouvertement politiques, comme dans la feuille consacrée à la dénonciation De la secte des trembleurs (1788), où l'on peut lire que ces Polonais modérés, face à la menace étrangère, « croient que le meilleur moyen d'empêcher qu'on entre chez eux est de tenir leurs portes ouvertes, parce qu'ils disent que s'ils les fermaient, cela pourrait donner à quelqu'un l'envie de les ouvrir » (Euvres, III, p. 266).
} 
alors qu'il vient de prétendre que ses «parents ne négligèrent rien pour [lui] donner une éducation excellente », il lui répond : «Taisez-vous, ma fille, ce que vous dites-là, je le dirai à la fin, mais il faut toujours commencer une histoire comme j'ai fait » (II, 47).

Au-delà de l'impératif communicationnel du storytelling ${ }^{13}$, tout se passe comme s'il importait peu, en démocratie, que je croie à ce que je dis ou que vous y croyiez en m'écoutant : ce que je vous demande, c'est d' «écoute[r] des choses que vous savez déjà, ce sera le moyen de ne point ignorer ce qui vous est connu dès longtemps » (II, 46); si l'adéquation de mes paroles à mes croyances est un problème secondaire, en revanche "il est essentiel que je prouve que j'ai toujours été démocrate, $\&$ dès avant la naissance des plus vieux députés » (II, 47).

Autant dire que la démocratie est, du point de vue rhétorique, le règne du politiquement correct : ce qui compte n'est pas de dire ce qu'on pense (ou ce qui est vrai), mais de dire ce qu'il faut dire (et de ne pas dire ce qu'on ne saurait entendre). C'est tout le discours de Cassandre qui apparaît ainsi comme destiné à faire parade d'un démocratisme au-dessus de tout soupçon : c'est parce qu'il faut avoir reçu une excellente éducation qu'il commence son histoire en y faisant référence, c'est pour avoir l'occasion de faire l'éloge de « ce bon peuple que ses ennemis n'ont point cessé de calomnier » qu'il raconte l'histoire de sa fromagerie - et c'est surtout pour "prouver qu'il a toujours été démocrate » (plutôt que pour exprimer une sensibilité personnelle) qu'il fait mine de se préoccuper du «sang des malheureux » Africains qui arrose l'or ramené d'Amérique. La démocratie valorise un espace où la parole doit se conformer à ce qu'on dit suivant les modes et les engouements du moment («je préférai le commerce, qui est comme l'on dit l'âme des états commerçants », II, 47 ; «l'on avait bien raison de dire que rien ne serait plus fatal qu'une contre-révolution », II, 51).

Ici encore, la démocratie paraît reposer sur une convergence de convenances : de même que les gouvernés obéissent aux commandements qui conviennent à leurs intérêts (sans quoi ils sont conduits à se débarrasser des gouvernants qui ne leur conviendraient plus), de même chacun se sent-il appelé à dire ce qu'il convient de dire en fonction des conventions langagières et idéologiques du moment. S'il est à la mode de se vanter d'une éducation excellente, de chanter l'éloge du bon peuple ou de faire déclaration d'anti-esclavagisme, je sacrifierai à ces rites, non pour exprimer mon opinion intime, mais pour assurer ma bonne insertion dans le réseau de communication démocratique. Au sein d'une collectivité qui se flatte de son égalité et de son indistinction, il devient effectivement « essentiel » de garantir et d'alimenter constamment son appartenance au réseau socialisant. Chaque parole a la fonction d'un passeport ou d'une patte blanche destinés à prouver qu'on est bien à sa place au sein de cette communauté d'égaux et de semblables : le comique de déplacement lexical sur lequel repose la parade tient précisément à ce que les termes que j'emploie doivent servir à la fois de moyens de référence à une certaine portion du réel que j'ai besoin de désigner et de mots de passe assurant le renouvellement de mon accès à la communauté de semblables.

\section{Le régime rhétorique de gouvernementalité démocratique impose une structure de} communication circulaire qui fait de tout échange conversationnel un spectacle adressé en réalité à un tiers caché (le public).

S'il est tellement essentiel de dire ce qu'on dit, c'est dans la mesure où chacun sait qu'on l'écoute. Ce que la pièce de Potocki met en lumière, c'est qu'en démocratie, toute parole est marquée par une structure de double adresse : au-delà de l'interlocuteur particulier qu'elle paraît viser explicitement (un père parle à sa fille), elle est en réalité orientée vers une

\footnotetext{
${ }^{13}$ Sur l'importance prise par le storytelling dans la communication politique contemporaine, voir par exemple Christian Salmon, Storytelling. La machine à fabriquer les histoires et à formater les esprits, Paris, La Découverte, 2007 et Francesca Polletta, It Was Like a Fever: Storytelling in Protest and Politics, Chicago, University of Chicago Press, 2006.
} 
instance tierce (le public), qui correspond exactement à ce «on» collectif servant à la fois de destinateur premier et de destinataire ultime à l'acte de parole. Cette structure relève donc d'une forme de communication essentiellement circulaire, à travers laquelle la communauté de semblables se reproduit elle-même par l'entremise des effets de similitude et de résonances qu'elle induit dans les propos de chacun de ses membres. C'est la bizarrerie de cette structure de communication que font sentir les deux premières tirades de la parade :

CASSANDRE : Je suis seul, profitons de ce monologue favorable pour faire venir ma fille, \& lui faire la motion d'épouser un mari de ma main, qui sera l'époux que je lui destine. $[\ldots]$

ZERZABELLE: Me voici mon père, \& je n'étais pas bien loin, car voyant que vous parliez seul \& si haut que les sourds pouvaient vous entendre, je me suis approchée pour écouter \& c'est z'ainsi que j'ai appris l'époux et les cent coups de pieds que vous voulez me donner dans le ventre $[\ldots]$.

CASSANDRE : Ma fille, ne vous emportez point : il est vrai que j'ai parlé fort haut, mais j'avais mes raisons pour cela, \& je dois parler encore de la même manière, car l'exposition n'est pas finie. (I \& II, 46).

Tout un pan de l'humour de la pièce tient à sa dimension méta-théâtrale. Conformément à la loi du genre, la parade tend à exploiter de façon parodique les propriétés de la représentation dramatique : ses tréteaux provisoires offrent une occasion de se moquer des conventions qui régissent la scène du théâtre officiel. Cassandre n'est pas présenté comme un père cherchant à marier sa fille, mais comme un acteur qui s'adresse à un public en incarnant le personnage d'un père parlant à sa fille - à savoir comme un bateleur qui profite d'un «monologue favorable » pour se livrer au travail «d'exposition » devant planter la situation de toute pièce de théâtre.

Telle est bien la structure de communication à double adresse évoquée ci-dessus : le personnage/acteur fait mine de s'adresser à un autre personnage/acteur, mais vise en réalité un tiers caché, le public d'un «on» multiple et indistinct qui l'entend depuis la salle. Le dispositif théâtral et conceptuel de Cassandre démocrate nous invite donc à reconnaître en la démocratie la structure d'une société du spectacle: l'agent politique doit avant tout se comporter comme un acteur, qui joue aussi bien que possible le rôle dont il veut projeter l'image parmi ses concitoyens, disant ce qu'il faut dire pour être (ré-)élu et pour pousser ses spectateurs/électeurs à partager sa vision de la prospérité nationale. Il se sait être à chaque instant sur la scène publique, observé et écouté, applaudi ou hué selon ce qu'on dira de ce qu'il a pu dire ou faire. Quel que soit son environnement immédiat, il parle toujours « seul \& si haut que les sourds puissent l'entendre », dans la mesure où ses interlocuteurs immédiats ne sont que des excuses ou des médiations pour valoriser l'image qu'il souhaite répandre de lui au sein du public de la communauté des semblables.

Cassandre se comporte donc en parfait (politicien) démocrate lorsqu'il tourne finalement à son avantage public l'humiliation privée que lui a imposée la bastonnade de Léandre : en donnant sa fille à l'aristocrate qu'il maudissait quelques minutes plus tôt, et en le faisant renoncer à ses coups contre-révolutionnaires, il pense avoir trouvé « une occasion de sauver la France, \& de mériter une couronne civique, qu['il] ne manquera pas de faire mettre dans les Gazettes » $(\mathrm{V}, 51)$. Comme on peut s'en assurer quotidiennement en regardant les nouvelles de 20 heures, ce qui compte dans nos démocraties médiatiques n'est pas tant l'action performée par le politicien que la façon dont cette action est perçue et reflétée par les journaux, les commentateurs et les sondages. Selon le renversement déjà abondamment décrit par Guy Debord, Paul Virilio et Jean Baudrillard, «la réalité » n'est pas ce qui se passe actuellement dans les rues, les usines, les bureaux ou les parlements, mais ce qui passe à la télévision et dans les médias les plus en vue : 30000 personnes qui manifestent dans une de 
nos villes sans passer au Journal de 20 heures comptent infiniment moins, du point de vue de notre médiocratie politique, que 300 activistes qui parviennent à attirer sur leur cause une demi-minute d'attention télévisuelle et la page de titre d'une gazette nationale.

Pour discret qu'il soit, le clin d'œil qu'adresse Cassandre démocrate au rôle de la presse et aux phénomènes de spectacularisation s'inscrit dans des séries récurrentes qui traversent toute l'œuvre et toute la pensée de Potocki. Outre ses expériences personnelles de directeur de gazettes, destinées elles aussi à distribuer des couronnes civiques aux acteurs du mouvement patriotique polonais, c'est toute la macrostructure du Manuscrit trouvé à Saragosse qui décrit une micro-société du spectacle établie au sein de la Sierra Morena, où elle attire deux voyageurs-pigeons (Alphonse van Worden et Pèdre Velasquez) qu'elle manipule au sein d'une vaste et parfaite mise en scène. Le destin de la dynastie et de la conspiration des Gomelez - et, à travers eux, celui d'un projet de monarchie universelle - se trouve suspendu à la réussite d'un spectacle où les agents s'avèrent finalement n'avoir été que des acteurs, dont les échanges ne s'adressaient en réalité qu'au public constitué par les deux voyageurs ${ }^{14}$. Quoiqu'à travers des genres et sur des modes très différents, la parade et le roman décrivent bien une société que traverse et structure de part en part une logique du spectacle, au sein de laquelle le théâtre ne se contente pas de représenter la vie, mais s'avère donner la raison de sa constitution sociale.

9. Le régime rhétorique de gouvernementalité démocratique répond exactement à la structure de la parade en ce que le débat politique y fonctionne comme un préambule de nature publicitaire destiné à capter l'attention et à fourguer un billet d'adhésion à un public prêt à faire un pari sur sa satisfaction à venir.

Au sein d'une telle société du spectacle, le jeu politique de la démocratie remplit une fonction remarquablement similaire à celle qui définissait la parade sur les lieux de foire des $\mathrm{XVII}^{\mathrm{e}}$ et $\mathrm{XVIII}^{\mathrm{e}}$ siècles. Avant de se constituer en genre propre et de servir à l'amusement des classes supérieures, la parade servait en effet à appâter le chaland en lui donnant un avantgoût gratuit de ce dont il pourrait avoir l'expérience réelle en achetant un billet pour le spectacle complet. Conformément à sa vocation méta-théâtrale, la parade de Potocki met en scène ce geste de captation d'attention et d'affects dans ses premières et dans ses dernières répliques : on a vu Cassandre insister initialement pour que sa fille, qui s'était "approchée pour écouter », «prenne une chaise afin d'être assise plus commodément » (pour l'entendre dire des choses qu'elle sait déjà), et on a vu Léandre reconnaître que ce sont « les appâts » de Zerzabelle qui lui ont rendu irrésistible une mésalliance qu'il «ne devrait pas seulement prendre avec des pincettes $» . .$.

Or cette fonction publicitaire de la parade, dans toute sa dimension d'appât, informe également le jeu de la démocratie parlementaire, qui s'éclaire de couleurs plus réalistes dès lors qu'on l'envisage à travers l'imaginaire de la foire plutôt qu'à travers celui du forum. À quoi sert donc un discours politique, sinon à tenter d'amener des électeurs à «acheter» (to buy) un argumentaire, en investissant leur bulletin de vote dans un programme dont les promesses de félicité leur semblent crédibles ? Dans la foire aux idées et aux mots d'ordre, il s'agit de savoir attirer l'attention des passants, capter leurs affects, mobiliser leurs craintes et

\footnotetext{
${ }^{14}$ Pour une interprétation du Manuscrit essayant de prendre la mesure de cette dimension spectaculaire, je renvoie à mes articles «L'imprimerie des Lumières: filiations de philosophes dans le Manuscrit trouvé à Saragosse de Jean Potocki », in Pierre Hartmann et Florence Lotterie (éd.), Le Philosophe romanesque. L'image du philosophe dans le roman des Lumières, Presses universitaires de Strasbourg, 2007, p. 301-335 ; «Potocki and the Spectre of the Postmodern », Comparative Criticism, ${ }^{\circ}$ 24, automne 2002, Cambridge University Press, pp. 141-165 ; et «Le Manuscrit trouvé à Saragosse : une machine à tordre le droit », à paraitre dans François Rosset et Dominique Triaire (éd.), Jean Potocki ou le Dédale des Lumières.
} 
leurs espoirs - sur l'escompte d'un résultat qui ne peut être qu'à venir (dans les quatre ou cinq ans du rythme électoral).

Face à cette structure de parade que revêt la démocratie telle que l'esquisse Potocki, on comprend pourquoi le politicien Cassandre, à chaque fois que sa fille pointe un mensonge grossier dans ses propos et le rappelle à la vérité travestie, rétorque invariablement : «je le dirai à la fin » (II, 47-48). En régime de démocratie parlementaire, la parole politique a la forme d'une promesse de prospérité faisant l'objet d'un pari sur l'avenir - promesse vouée à ne rencontrer sa vérité (satisfaction ou, plus généralement, déception) qu'à la fin du mandat pour lequel le candidat aura été élu. Le rythme de la vie politique est ainsi marqué par un délai constitutif, par une prospérité qui apparaît comme constamment différée ou par une «crise» (relative au chômage, à la dette publique, au pouvoir d'achat) dont on voit perpétuellement « le bout du tunnel », mais dont on n'a jamais l'impression de sortir vraiment - puisqu'on ne fait en réalité périodiquement que changer d'objet de promesse ou de crainte, sans jamais en accomplir ou en liquider aucun.

On entrevoit aussi par là même une signification possible du petit échange au cours duquel Cassandre vante les mérites de sa fille en la décrivant comme un Phénix:

CASSANDRE : Votre mère me fut enlevée par un sort funeste, $\&$ dès lors je me consacrai à votre éducation, vous répondîtes à mes soins \& devîntes un phénix.

ZERZABELLE: Ah! mon père, vos entrailles me regardent avec une indulgence maternelle : les phénix \& moi, nous ne passons pas souvent par la même porte.

CASSANDRE : Point de fausse modestie ma fille, je me connais en phénix \& vous en êtes un sur ma parole, mais ne m'interrompez plus. (II, 48)

Là où le principe abstrait de la démocratie parlementaire prévoit la remise des compteurs à zéro au terme de chaque calendrier électoral, la visée du politicien démocrate est de voir son électorat renaître de ses cendres à la fin de son mandat - même si une forte tendance à l'alternance fait que le public et les phénix ne passent pas souvent par la même porte. Face à une logique qui tend fortement à éjecter les sortants, et voyant son image vouée à être descendue en flammes par ses opposants, le politicien sait que sa survie repose sur une capacité de renaissance périodique (de mère en fille), au fil des promesses et des crises autour desquelles il sera parvenu à mobiliser le débat public pour chaque (ré)génération électorale successive.

En mettant dans la bouche de Cassandre un discours non seulement absurde mais ennuyeux, qui a la vertu de faire s'endormir sa fille assise pour l'écouter (II, 47), la parade représente dès lors la hantise suprême de tout bateleur-politicien: échouer à captiver l'attention du public. Alors que durant les deux derniers siècles, nos économies modernes se sont mobilisées pour combattre la rareté des biens matériels (nourriture, vêtements, logements, voitures, etc.), les développements parallèles des communications et du temps libre permis par l'abondance de biens de consommation fait apparaître une nouvelle rareté qui en arrive à ré-organiser aussi bien nos logiques économiques que nos espaces politiques : le temps d'attention. L'hyperactivité de certains présidents témoigne d'une conscience lucide et opportuniste de cette nouvelle économie de l'attention ${ }^{15}$, au sein de laquelle faire parler de soi (en bien ou en mal), c'est déjà avoir gagné la première bataille pour la survie politique - rien n'étant pire que l'indifférence de média et le sommeil du public (ne serait-ce que parce que le choc du réveil risque d'être imprévisible). Qu'il s'agisse de vendre (to sell) la marque d'un soda, d'une voiture, d'un parfum, le slogan d'un parti ou le visage radieux d'un candidat, les

\footnotetext{
${ }^{15}$ Voir sur ce point l'introduction et le premier chapitre de Richard A. Lanhan, The Economics of Attention. Style and Substance in the Age of Information, Chicago, University of Chicago Press, 2006 et Maurizio Lazzarato, Les révolutions du capitalisme, Paris, Les empêcheurs de penser en rond, 2004.
} 
experts de la communication s'ingénient - comme les auteurs de parades - à appâter le chaland par une parole dont l'essence est publicitaire, dans la mesure où son objet est indissociablement de mobiliser l'attention du public et d'infléchir ses gestes d'adhésion à venir.

La pièce suggère aussi, du même coup, que - contrairement à l'infamie dont fait généralement objet l'abstentionnisme - le refus de prêter attention au jeu politique (rester chez soi le jour du vote, éteindre sa télévision durant les grands débats de la foire électorale) peut constituer à la fois une ultime forme de rejet et une toute première forme résistance à la perversion du jeu politicien: s'endormir durant les discours de nos divers Cassandre démocrates peut nous donner l'occasion de rêver à ces «autres mondes possibles » que leurs coq-à-l'âne faussement réalistes étouffent dans l'œuf tant que nous restons enfermés dans les fausses alternatives (promesses et crises) dont leurs argumentaires nous obnubilent.

La vision de la démocratie esquissée par cette parade de Potocki est donc certes critique dans la mesure où elle dépeint un monde nourri de mensonges, d'âneries, d'opportunisme, de vantardise et de violence sous-jacente. On ne saurait toutefois dire qu'une telle piécette invite à condamner la démocratie comme telle, ne serait-ce que parce que l'aristocrate Léandre donne une image aussi ridicule d'une possible contre-révolution nobiliaire que le démocrate Cassandre donne une image peu flatteuse de la Révolution bourgeoise. L'impression qui reste à l'esprit, au terme des quatre dernières répliques que se partagent Cassandre, Gile et Léandre, est celle d'une assez joyeuse et finalement bien innocente plaisanterie dont le happy end est aussi finement grinçant que l'est le reste de la pièce, sous ses faux airs de vulgarité. Dès lors que «la contre-révolution n'est plus à craindre », comme en convient la tirade finale de Léandre, le promis annonce à sa promise que «notre bonheur z'est assuré » et qu' «il ne nous reste plus qu'à vaquer aux embarras de votre félicité » $(\mathrm{V}, 52)$. Conformément aux lois de la parade, tout paraît se résoudre pour le mieux dans le plus démocratique des mondes: Zerzabelle a pu écarter le mari qui suscitait sa répugnance pour obtenir celui qui convenait à ses désirs ; Léandre, tout occupé à assurer son bonheur, ne songe plus à passer son épée au travers de l'assemblée nationale; Gile pourra continuer à vendre ses fromages (sitôt que la tourmente révolutionnaire aura permis une reprise du commerce); et Cassandre pourra se glorifier dans les gazettes d'une couronne civique obtenue pour avoir sauvé la France.

Avec deux siècles de distance - à la suite de l'invention de la machine à vapeur, du chemin de fer, du télégraphe, de la télévision, des centrales nucléaires, du Boeing 747, du lave-vaisselle, du Hummer et du Blackberry, et avec le recul que donnent les caps passés en 1794, 1848, 1945, 1968, 1989 et 2001 - nos modes de vie individuelle et nos régimes de démocratie politique paraissent à la fois «avoir z'assuré notre bonheur » et risquer de nous écraser sous «les embarras de notre félicité ». De même que le Manuscrit trouvé à Saragosse se conclut sur un partage de richesses émancipées de tout prosélytisme fanatique, mais également vidées de toute finalité autre que celle d'une jouissance désœuvrée, de même Cassandre démocrate laisse-t-il son audience (amusée) suspendue à un événement menaçant qui «n'a pu s'effectuer» (la contre-révolution), mais dont l'absence fait planer un vague sentiment d'inanité et de déréliction. À nous sans doute de rêver comment, dès lors que «la contre-révolution n'est plus à craindre », une autre révolution - plus réellement démocratique - pourra enfin devenir possible.

On pourrait commencer par imaginer une autre parade qui se recentrerait autour de la fille de Cassandre (à laquelle la scène finale de la pièce de Potocki ne donne pas la parole), anoblie par son mariage à Léandre dans un régime ne reconnaissant plus aucune noblesse attitrée (Zerzabelle démocrate ? $^{16}$ ). On pourrait voir en Zerzabelle non seulement, comme

\footnotetext{
${ }^{16}$ Un tel devenir-femme de la démocratie aurait bien entendu fait rire Potocki, qui se moque des discussions d'un club révolutionnaire visant à « répandre d'absurdes paradoxes tels que le legem agrariam ou partage égal des
} 
dans la pièce jouée à Łańcut, une figure de résistance du public, fort de son bon sens pratique, contre les coq-à-l'âne et les mauvais mariages qu'essaient de lui fourguer les divers marchands de fromage qui se sont accaparé le pouvoir économique et politique durant la modernité bourgeoise, mais l'appel à une nouvelle noblesse - déjà réclamée à l'époque par une auteure comme Isabelle de Charrière - qui mettrait au rancart le privilège du sang pour cultiver la distinction d'une éducation (véritablement) excellente (quoique universelle), qui renoncerait à l'esprit de clan pour s'adonner à la curiosité anthropologique des voyages lointains, qui troquerait l'épée et le point d'honneur contre la plume et l'imagination romanesque pour faire de la créativité artistique et intellectuelle le plus grand mérite d'une aristocratie démocratisée. Le scénario d'une telle pièce consacrée au devenir-artiste d'une nouvelle noblesse esquisserait une double figure qui nous est finalement familière, celle d'un officier (aux Gardes wallonnes) devenu érudit, d'un patriote (polonais) devenu anthropologue et d'un comte devenu conteur (Alphonse Potocki démocrate?).

terres, l'admission des femmes au gouvernement et autres rêveries de ce genre » (Lettre du 23 décembre 1793 à Séverin Potocki in Euvres, v, p. 26). 